POS $\quad \begin{aligned} & \text { PROCEEDINGS } \\ & \text { OF SCIENCE }\end{aligned}$

\title{
Complete gauge invariant decomposition of the nucleon spin
}

\author{
Yoshitaka Hatta* \\ Yukawa institute for theoretical physics, Kyoto University, Kyoto 606-8502, Japan \\ E-mail: hatta@yukawa.kyoto-u.ac.jp
}

We overview the recently achieved complete decomposition of the nucleon spin. This is the gauge invariant completion of the Jaffe-Manohar sum rule, and involves twist-three GPDs. Its precise relation to the Ji sum rule is clarified.

XV International Conference on Hadron Spectroscopy-Hadron 2013 4-8 November 2013

Nara, Japan

${ }^{*}$ Speaker. 


\section{Introduction}

The facts that the nucleon has spin one-half and that it is not an elementary particle open up a fundamental research field examining how the total nucleon spin is accounted for by quarks' and gluons' degrees of freedom. In recent years there has been renewed interest in this old problem which has resulted in important new findings. The purpose of this talk is to briefly overview these developments, but before doing so, let me first review the status before 2008. Two decomposition schemes, each of which has its own pros and cons, have been known for a long time:

1. Jaffe-Manohar sum rule [1]

$$
\frac{1}{2}=\frac{1}{2} \Delta \Sigma+\Delta G+L_{c a n}^{q}+L_{c a n}^{g} .
$$

This is based on the decomposition of the canonical energy/angular momentum tensor in QCD. It is a complete decomposition in the sense that the right hand side contains four terms corresponding to the spin and the orbital angular momentum (OAM) of quarks and gluons. However, it is not a gauge invariant decomposition (excepting the quark spin $\Delta \Sigma$ ). The default gauge is the light-cone gauge which allows a partonic interpretation for each component.

2. Ji sum rule [2]

$$
J^{q}=\frac{1}{2} \int_{-1}^{1} d x x\left(H_{q}(x)+E_{q}(x)\right), \quad J^{g}=\frac{1}{4} \int_{-1}^{1} d x\left(H_{g}(x)+E_{g}(x)\right) .
$$

This relates the quark/gluon contribution $\left(J^{q}+J^{g}=\frac{1}{2}\right)$ to the quark/gluon twist-two generalized parton distributions (GPDs). It is based on the so-called improved energy/angular momentum tensor of QCD, and as such, all the operators involved are local and gauge invariant. Their matrix elements (i.e., GPDs) are measurable experimentally from deeply-virtual Compton scattering (DVCS). $J^{q}$ (but not $J^{g}$ ) can be further decomposed, gauge invariantly, into the spin and the OAM parts: $J^{q}=\frac{1}{2} \Delta \Sigma+L^{q}$. This kinetic OAM $L^{q}$ is different from the canonical OAM $L_{c a n}^{q}$ in (1.1), and does not satisfy the commutation relation $\left[\vec{L}^{q} \times \vec{L}^{q}\right] \neq i \vec{L}^{q}$.

There have been enormous experimental effort to independently measure $\Delta G$ and GPDs. However, it is not clear how to combine the outcomes of these measurements because they are motivated by different decomposition schemes, and no one knew the precise relation between the two schemes.

\section{Complete gauge invariant decomposition}

In 2008, Chen et al. suggested a new approach towards achieving a complete, gauge invariant decomposition scheme [3] (see also, [4]). This has triggered a great deal of debate and confusion among experts. After the dust has settled, the following decomposition has emerged

$$
\begin{aligned}
& M_{\text {quark-spin }}^{\mu \nu \lambda}=-\frac{1}{2} \varepsilon^{\mu \nu \lambda \sigma} \bar{\psi} \gamma_{5} \gamma_{\sigma} \psi, \quad M_{\text {quark-orbit }}^{\mu \nu \lambda}=\bar{\psi} \gamma^{\mu}\left(x^{v} i D_{\text {pure }}^{\lambda}-x^{\lambda} i D_{\text {pure }}^{v}\right) \psi \\
& M_{\text {gluon-spin }}^{\mu \nu \lambda}=F_{a}^{\mu \lambda} A_{\text {phys }}^{v a}-F_{a}^{\mu v} A_{\text {phys }}^{\lambda a}, \quad M_{\text {gluon-orbit }}^{\mu \nu \lambda}=-F_{a}^{\mu \alpha}\left(x^{\nu}\left(D_{\text {pure }}^{\lambda} A_{\alpha}^{\text {phys }}\right)_{a}-x^{\lambda}\left(D_{\text {pure }}^{v} A_{\alpha}^{\text {phys }}\right)_{a}\right) .
\end{aligned}
$$


where [5]

$$
A_{\text {phys }} \equiv \frac{1}{D^{+}} F^{+\mu}, \quad D_{\text {pure }}^{\mu} \equiv D^{\mu}-i g A_{\text {phys }}^{\mu} .
$$

(2.1) is written in terms of the angular momentum tensor, and can be recast in a similar form as in (1.1) after taking the matrix element $\left\langle P S\left|M^{+i j}\right| P S\right\rangle$. The result is essentially the gauge invariant completion of the Jaffe-Manohar scheme (1.1). (It reduces to Jaffe-Manohar in the light-cone gauge $A^{+}=0$.) The gluon spin part agrees with $\Delta G$, and moreover it provides the precise gauge invariant definitions of the quark and gluon canonical OAMs $L_{c a n}^{q, g}$.

\section{Orbital angular momentum}

By definition, the canonical OAM $L_{c a n}^{q}$ satisfies the commutation relation while the kinetic OAM $L^{q}$ does not. One way to characterize the two types of OAMs is through the Wigner distribution

$$
W[x, q]=\int \frac{d^{4} z}{(2 \pi)^{4}} e^{i k \cdot z} \bar{\psi}\left(x-\frac{z}{2}\right) \mathscr{W} \psi\left(x+\frac{z}{2}\right) .
$$

Since the Wigner distribution contains information of both position $x$ and momentum $q$, it is natural to define an OAM by forming the cross product $\vec{x} \times \vec{q}$ and averaging it with the weight (3.1). This was first considered in [6], but the Wilson line $\mathscr{W}$, which renders the nonlocal operator in (3.1) gauge invariant, was neglected. It turns out that the nature of the resulting OAM does depend on the choice of the Wilson. If one uses a Wilson line which goes to light-cone infinity $\left(x^{-}= \pm \infty\right)$ and then comes back, one gets the canonical OAM $L_{c a n}^{q}$ [7]. However, if one uses the straight Wilson line (from $x-z / 2$ to $x+z / 2$ ), one rather gets the kinetic OAM $L^{q}[8]$.

\section{Twist analysis}

Now we come to the relation between the complete decomposition and the Ji decomposition

$$
J^{q}=\frac{1}{2} \Delta \Sigma+L_{c a n}^{q}+L_{p o t}, \quad J^{g}+L_{p o t}=\Delta G+L_{c a n}^{g},
$$

where $L^{q}-L_{c a n}^{q} \equiv L_{p o t}$ is called the potential angular momentum. Remarkably, these relations can be understood at the density level [9]. Indeed, it is possible to uniquely (in a certain sense) define the density of the canonical OAM $L_{c a n}^{q, g}=\int d x L_{c a n}^{q, g}(x)$. This allows us to analyze the twist structure of the complete decomposition, and in particular, its relevance to twist-three GPDs.

For this purpose, we define the following twist-three quark-gluon correlator

$$
\begin{aligned}
& \int \frac{d y^{-} d z^{-}}{(2 \pi)^{2}} e^{\frac{i}{2}\left(x_{1}+x_{2}\right) \bar{P}^{+} z^{-}+i\left(x_{2}-x_{1}\right) \bar{P}^{+} y^{-}}\left\langle P^{\prime} S^{\prime}\left|\bar{\psi}\left(-z^{-} / 2\right) \gamma^{+} \mathscr{W}_{\frac{-z}{2} y} g F^{\mu v}\left(y^{-}\right) \mathscr{W}_{y \frac{z}{2}} \psi\left(z^{-} / 2\right)\right| P S\right\rangle \\
& =\frac{1}{\bar{P}^{+}} \varepsilon^{\mu v \rho \sigma} \bar{S}_{\rho} \Delta_{\sigma} \Phi_{F}\left(x_{1}, x_{2}\right)+\cdots .
\end{aligned}
$$

This is similar to the matrix element that generates the (transverse) single-spin asymmetry, but here it is a nonforward matrix element $\left(\Delta=P^{\prime}-P\right)$ and we have in mind the longitudinal polarization. It is easy to show that [7]

$$
L_{p o t}=\int d x_{1} d x_{2} \mathscr{P} \frac{\Phi_{F}\left(x_{1}, x_{2}\right)}{x_{1}-x_{2}} .
$$


In addition to the 'F-type' correlator (4.2), let us similarly define the 'D-type' correlators

$$
\begin{aligned}
F . T .\left\langle P^{\prime} S^{\prime}\left|\bar{\psi} \gamma^{+} F^{+i} \psi\right| P S\right\rangle & \sim \Phi_{F}\left(x_{1}, x_{2}\right), \\
\text { F.T. }\left\langle P^{\prime} S^{\prime}\left|\bar{\psi} \gamma^{+} \gamma_{5} F^{+i} \psi\right| P S\right\rangle & \sim \widetilde{\Phi}_{F}\left(x_{1}, x_{2}\right), \\
F . T .\left\langle P^{\prime} S^{\prime}\left|\bar{\psi} \gamma^{+} D^{i} \psi\right| P S\right\rangle & \sim \Phi_{D}\left(x_{1}, x_{2}\right), \\
\text { F.T. }\left\langle P^{\prime} S^{\prime}\left|\bar{\psi} \gamma^{+} \gamma_{5} D^{i} \psi\right| P S\right\rangle & \sim \widetilde{\Phi}_{D}\left(x_{1}, x_{2}\right),
\end{aligned}
$$

It is known that generally these two types of correlators are related [10]. In the present case, the relation reads

$$
\Phi_{D}\left(x_{1}, x_{2}\right)=\delta\left(x_{1}-x_{2}\right) L_{c a n}^{q}\left(x_{1}\right)+\mathscr{P} \frac{1}{x_{1}-x_{2}} \Phi_{F}\left(x_{1}, x_{2}\right),
$$

which is the doubly-unintegrated version of $L^{q}=L_{c a n}^{q}+L_{\text {pot }}$ (cf. (4.3)). Eq. (4.5) naturally defines the canonical OAM density $L_{c a n}^{q}=\int d x L_{c a n}^{q}(x)$. The delta function $\delta\left(x_{1}-x_{2}\right)$ ensures that, in the quark-gluon-quark system described by the operator $\bar{\psi} \gamma D \psi$, the gluon carries zero longitudinal momentum $x_{2}-x_{1}=0$. Thus the variable $x$ in $L_{c a n}^{q}(x)$ is indeed the longitudinal momentum fraction assigned to the quark, which makes its density interpretation possible. In contrast, there is ambiguity in defining a 'density of the kinetic OAM' $L^{q}=\int d x L^{q}(x)$. For instance, one can define either $L^{q}(x)=\int d x^{\prime} \Phi_{D}\left(x, x^{\prime}\right)$ or $L^{q}(x)=\int d x^{\prime} \Phi_{D}\left(x+x^{\prime} / 2, x-x^{\prime} / 2\right)$.

The operator representation of $L_{\text {can }}^{q}(x)$ is rather complicated, but owing to the equation of motion it can be written as [9]

$$
\begin{aligned}
& L_{c a n}^{q}(x)=x\left(H_{q}(x)+E_{q}(x)+G_{3}(x)\right)-\Delta q(x) \\
& \quad-\int d x^{\prime} \mathscr{P} \frac{1}{x-x^{\prime}}\left(\Phi_{F}\left(x, x^{\prime}\right)+\widetilde{\Phi}_{F}\left(x, x^{\prime}\right)\right),
\end{aligned}
$$

where $\Delta q$ is the usual polarized quark distribution and $G_{3}(x)$ is one of the twist-three GPDs defined as

$$
\text { F.T. }\left\langle P^{\prime} S^{\prime}\left|\bar{\psi}(-z / 2) \gamma_{\perp}^{i} \psi(z / 2)\right| P S\right\rangle=G_{3}(x) \bar{u}\left(P^{\prime} S^{\prime}\right) \gamma_{\perp}^{i} u(P S)+\cdots .
$$

Integrating (4.6) over $x$, we get

$$
\int d x x G_{3}(x)=-L^{q}
$$

This identity was first derived in Ref. [11]. However, there the authors worked in the parton model where there is no distinction between $L^{q}$ and $L_{c a n}^{q}$. We see that (4.8) is valid in full QCD as well. (4.6) and (4.8) show that, while the integral of $G_{3}$ is related to the kinetic OAM, $G_{3}(x)$ itself is rather related to the canonical OAM density.

Furthermore, $G_{3}(x)$ can be eliminated from (4.6) due again to the equation of motion. The result is

$$
\begin{aligned}
L_{c a n}^{q}(x)=x \int_{x}^{\varepsilon(x)} & \frac{d x^{\prime}}{x^{\prime}}\left(H_{q}\left(x^{\prime}\right)+E_{q}\left(x^{\prime}\right)\right)-x \int_{x}^{\mathcal{E}(x)} \frac{d x^{\prime}}{x^{\prime 2}} \Delta q\left(x^{\prime}\right) \\
& -x \int_{x}^{\varepsilon(x)} d x_{1} \int_{-1}^{1} d x_{2} \Phi_{F}\left(x_{1}, x_{2}\right) \mathscr{P} \frac{3 x_{1}-x_{2}}{x_{1}^{2}\left(x_{1}-x_{2}\right)^{2}} \\
& -x \int_{x}^{\varepsilon(x)} d x_{1} \int_{-1}^{1} d x_{2} \widetilde{\Phi}_{F}\left(x_{1}, x_{2}\right) \mathscr{P} \frac{1}{x_{1}^{2}\left(x_{1}-x_{2}\right)} .
\end{aligned}
$$


Eq. (4.9) completely reveals the twist structure of $L_{c a n}^{q}(x)$. It can be decomposed into the 'WandzuraWilczek' (WW) part which is related to the twist-two GPDs, and the 'genuine twist-three' part (quark-gluon correlators). Taking the first moment of (4.9), we get

$$
L_{c a n}=J^{q}-\frac{1}{2} \Delta \Sigma-L_{p o t},
$$

which is precisely (4.1).

Similarly, we can define the canonical gluon OAM density $L_{c a n}^{g}(x)$ and analyze its twist structure. Again, the definition is unique in the sense that $x$ is interpretable as the longitudinal momentum fraction of the outgoing gluon. As in (4.6), the density is related to one of the twist-three gluon GPDs. By eliminating the twist-three GPD using the equation of motion, we get the decomposition of $L_{c a n}^{g}(x)$ into the WW part related to the twist-two gluon GPDs and genuine twist-three, three-gluon distributions. Its first moment of course coincides with (4.1).

In conclusion, we now have the complete gauge invariant decomposition of the nucleon spin even at the density level. It is the twist-three decomposition as compared to Ji's twist-two decomposition, and the relation between the two has been fully clarified.

\section{References}

[1] R. L. Jaffe and A. Manohar, Nucl. Phys. B 337, 509 (1990).

[2] X. Ji, Phys. Rev. Lett. 78, 610 (1997).

[3] X. -S. Chen, X. -F. Lu, W. -M. Sun, F. Wang and T. Goldman, Phys. Rev. Lett. 100, 232002 (2008).

[4] M. Wakamatsu, Phys. Rev. D 83, 014012 (2011)..

[5] Y. Hatta, Phys. Rev. D 84, 041701 (2011).

[6] C. Lorce and B. Pasquini, Phys. Rev. D 84, 014015 (2011).

[7] Y. Hatta, Phys. Lett. B 708, 186 (2012)..

[8] X. Ji, X. Xiong and F. Yuan, Phys. Rev. Lett. 109, 152005 (2012).

[9] Y. Hatta and S. Yoshida, JHEP 1210, 080 (2012)..

[10] H. Eguchi, Y. Koike and K. Tanaka, Nucl. Phys. B 752, 1 (2006) [hep-ph/0604003].

[11] M. Penttinen, M. V. Polyakov, A. G. Shuvaev and M. Strikman, Phys. Lett. B 491, 96 (2000). 